\title{
Avaliação do efeito da esplenectomia e auto-implante esplênico sobre algumas funções de monócitos em crianças com esquistossomose mansônica
}

\author{
Evaluation of the effect of splenectomy with autologous spleen tissue implantation in \\ some monocyte functions in children with hepatosplenic \\ schistosomiasis mansoni
}

\author{
Carlos T. Brandt ${ }^{1}$, Carlos Roberto C. Leite ${ }^{1}$, Raul Manhaes-de-Castro ${ }^{4}$, Carlos Brandt Filho ${ }^{3}$, \\ Francisco M. Manhaes-de-Castro ${ }^{3}$ e Celia Maria M. Barbosa-de-Castro ${ }^{2}$
}

\begin{abstract}
Adherence index, superoxide and TNF- $\alpha$ production in monocytes, with or without tuftsin treatment, were investigated in hepatosplenic schistosomiasis mansoni bearers with splenectomy with or without autologous implantation of spleen tissue. Three groups were evaluated: Healthy volunteers control group (CG) $(n=12)$; Splenectomy with seft auto-transplant AG $(\mathrm{n}=18)$ and Splenectomy without auto-transplant WAG $(\mathrm{n}=9)$. Adherence index and TNF- $\alpha$ did not differ among the groups. Superoxide production was similar in CG and AG, in the $1^{\text {st }}$ hour after cell stimulation. SP was larger in each hour time in $\mathrm{CG}$ and $\mathrm{AG}$ groups as compared WAS group. TT recovered normal pattern of SP in AG comparable with levels found in CG, with increase from the $1^{\text {st }}$ to $2^{\text {nd }}$ hour. However, TT did not alter SP in WAG, which remained reduced in all time points. Autologous implantation of spleen tissue seems to contribute for recovery and maintenance of the evaluated immunological reactions, which might be important in response to infections.
\end{abstract}

Key-words: Autologous implantation. Hepatosplenic schistosomiasis. Adherence index. Superoxide production. TNF- $\alpha$.

\section{RESUMO}

Investigamos em portadores de esquistossomose hepatoesplênica após esplenectomia com ou sem auto-implante esplênico: índice de aderência, produção de superóxido ( SP) e de TNF- $\alpha$ em monócitos, tratados ou não com tuftsina. Avaliamos três grupos: voluntários sadios CG (grupo controle) $(n=12)$; esplenectomizados com auto-implante $A G(n=18)$ e esplenectomizados sem auto-implante WAG $(\mathrm{n}=9)$. Índice de aderência e TNF- $\alpha$ não diferiram entre os grupos. SP foi semelhante em CG e AG na 1a hora após estimulação celular. SP foi maior em todos intervalos de tempo nos grupos CG e AG, comparados ao WAG. 0 tratamento com tuftsina recuperou o padrão de normalidade de SP em AG, com aumento da $1^{a}$ para a $2^{2}$ hora nos níveis do CG. 0 tratamento com tuftsina não alterou SP em WAG, permanecendo reduzida em todos intervalos. $\mathrm{O}$ auto-implante esplênico parece recuperar e manter os parâmetros imunológicos avaliados, que têm participação importante na resposta do hospedeiro às infecções.

Palavras-chaves: Auto-implante. Esquistossomose hepatoesplênica. Índice de aderência. Superóxido. Fator de necrose tumoral- $\alpha$.

\footnotetext{
1. Departamento de Cirurgia da Universidade Federal de Pernambuco, Recife, PE. 2. Departamento de Medicina Tropical e Laboratório de Imunopatologia KeizoAsami da Universidade Federal de Pernambuco, Recife, PE. 3. Faculdade de Medicina da Universidade Federal de Pernambuco, Recife, PE. 4. Departamento de Nutrição da Universidade Federal de Pernambuco, Recife, PE.

Financiamento: Conselho Nacional de Pesquisa - CNPq

Endereço para correspondência: Dra. Celia Maria Machado Barbosa de Castro. Laboratório de Imunopatologia Keizo-Asami ( LIKA)/UFPE. Campus Universitário, 50670-420 Recife, PE.

Tel.: 81 3271-8486, Fax: 81 3271-8485

e-mail: ccastro@ lika.ufpe.br

Recebido para publicação em 22/8/2003

Aceito em 1/11/2004
} 
0 Schistosoma mansoni infeta cerca de 200 milhões de pessoas no mundo, 120 milhões evoluem com sintomas e 20 milhões apresentam com dano hepatoesplênic $0^{13}$. No Nordeste do Brasil, a esquistossomose mansônica é hiperendêmica sendo a causa principal de hipertensão portal em crianças ${ }^{3}$. A maioria destas é tratada clinicamente, todavia, algumas requerem cirurgia, sendo a esplenectomia freqüente nesta região $0^{5}$. 0 baço é 0 maior órgão linfóide do organismo e principal local de resposta imune a antígenos presentes no sangue. A complicação mais temida é a sepse fulminante pós-esplenectomia, por bactérias encapsuladas, cuja mortalidade é de 50 a $75 \%{ }^{14}$. Isto tem sido atribuído à deficiência de fagócitos (neutróilos, monócitos e macrófagos), responsáveis pela destruição das bactérias ${ }^{25}$. 0 auto-implante de tecido esplênico no grande omento é a forma mais aceitável de preservar a função esplênica ${ }^{11}{ }^{17}$, reduzindo a sepse pósesplenectomia ${ }^{5}$.

Os fagócitos produzem citocinas pró-inflamatórias, particularmente, 0 Fator de Necrose Tumoral- $\alpha$ (TNF- $\alpha$ ) e ativa a resposta imune adaptativa ${ }^{6}$. A capacidade dos fagócitos em aderir in vitro a certos substratos é conhecida de longa data ${ }^{23}$. A adesão é a primeira etapa no processo fagocítico e é essencial para a resposta inflamatória ${ }^{25}$. Ademais, os fagócitos liberam derivados bactericidas do oxigênio, e 0 ânion superóxido $\left(\mathrm{O}_{2}\right.$. )$^{16}$. Indivíduos cujas células são incapazes de produzir $\mathrm{O}_{2}{ }^{-}$são altamente susceptíveis às infecções². Assim, 0 objetivo deste estudo foi avaliar estes aspectos funcionais dos monócitos em portadores de esquistossomose mansônica hepatoesplênica, submetidos à esplenectomia, ligadura da veia gástrica esquerda, com ou sem, auto-implante de tecido esplênico.

\section{MATERIAL E MÉTODOS}

Desenho do estudo e seleção dos pacientes. Foram incluídos no estudo 39 indivíduos com idade entre 15 e 31 anos, 19 do sexo masculino e 20 do feminino. Comparam-as, em ensaio clínico aberto, algumas funções de fagócitos em 3 grupos. 1. Grupo controle (CG)-constituído por 12 voluntários sadios com exame físico normal. 2. Grupo com esplenectomia e auto-implante (AG)-constituído por 18 adolescentes com esquistossomose hepatoesplênica, submetidos à esplenectomia, ligadura da veia gástrica esquerda e auto-implante de tecido esplênico no grande omento. 0 implante teve peso total de $100 \mathrm{~g} / \mathrm{baço}$. Realizouse cintigrafia com enxofre coloidal marcado com Tecnécio 99m, no pós-operatório para confirmação de tecido implantado funcionante. 3. Grupo com esplenectomia sem auto-implante (WAG) -constituído por 9 adultos jovens com esquistossomose hepatoesplênica, submetidos à desconexão ázigo-portal, no Serviço de Cirurgia Abdominal do Hospital das Clínicas da UFPE. A infecção pelo Schistosoma mansoni foi confirmada após 3 exames parasitológicos de fezes (técnica de Hofmann, Pons e Janer) colhidos em dias alternados. Todos foram submetidos previamente à dose única de oxaminiquine ( $20 \mathrm{mg} / \mathrm{Kg}$ de peso, via oral) e depois de 30 dias ao tratamento cirúrgico.
Obtenção de Monócitos do Sangue Periférico (PBMC). Foram coletados $18 \mathrm{~mL}$ de sangue com EDTA de todos os indivíduos, o sangue foi diluído 1:2 em meio de cultura RPMI 1640 e separado por gradiente de densidade em Ficoll. Em seguida, as células foram ressuspendidas em 2mLde RPMI 1640 com soro fetal bovino a 3\%, adicionado de antibióticos (penicilina 100U/mLe estreptomicina $100 \mathrm{mg} / \mathrm{mL}$ ). Após análise da viabilidade das células com azul tripan e contagem, foram incubadas em placas de 6 poços tipo Falcon (Becton\&Dickinson), dispensando-se $2 \mathrm{~mL}$ da suspensão com $10^{6}$ células $/ \mathrm{ml}$ por poço. A placa foi incubada por $1 \mathrm{~h}$, em atmosfera úmida, ${ }^{3}{ }^{\circ} \mathrm{C}, 5 \% \mathrm{CO}_{2}$ e em seguida, as células não aderentes foram contadas em um dos poços da placa.

Avaliação do índice de aderência nos monócitos. Alíquotas da suspensão de células não aderentes, após a primeira hora de cultura, foram adicionadas ao azul tripan e contadas em hemocitômetro. 0 IA foi calculado através da fórmula ${ }^{8} . \mathrm{IA}=100-\left(\mathrm{n} .{ }^{\circ}\right.$ de células não aderidas $/ \mathrm{ml}, \mathrm{n} .{ }^{\circ}$ inicial de macrófagos) x 100.

Dosagem do TNF- $\alpha$. Foi realizada pelo método ELISA com 0 kit (Quantikineâ M, R\&D Systems). Amostras de 100ml foram coletadas a partir do sobrenadante de cultura de monócitos estimulados por 6 horas com acetato miristato de forbol (PMA, Sigma).

Análise da liberação de superóxido $\left(\mathrm{O}_{2}^{-}\right) \cdot \mathrm{O}_{2}^{-}$foi induzido pela adição de PMA, em Hanks (HBSS, Gibco), na concentração de $2 \mathrm{ml} / \mathrm{mL}$. Foram preparados 2 sistemas de análise descontínua com avaliação a cada 1 hora, por 2 horas. A especificidade do ensaio foi garantida pela adição de superóxido dismutase ( SOD) de eritrócitos bovinos ( SIGMA - contendo $3000 \mathrm{u} / \mathrm{mg}$ de proteína em solução final de $3 \mathrm{mg} / \mathrm{mL}$ ). Para 0 preparo destes sistemas foram utilizados monócitos em cultura ( $2 \times 10^{6}$ células por $2 \mathrm{~mL}$ ), tratados ou não com tuftsina, com ou sem a presença de SOD. Os sistemas foram mantidos em incubadora a $37^{\circ} \mathrm{C}$, atmosfera úmida, $5 \%$ de $\mathrm{CO}_{2}$, por 10 minutos (ativação da SOD) . 0 ferrocitocromo c tipo IV de mitocôndria de coração de cavalo, (30mg/mL em HBSS, 2.4x 10-3 M, Sigma, USA) foi adicionado às células para quantificar a formação de $\mathrm{O}_{2}$ 'através da redução do ferricotocromo c. Amostras de $600 \mathrm{ml}$ foram retiradas de cada sistema, a 1 a alíquota recolhida, correspondia ao tempo "zero" de cada sistema e as amostras subseqüentes foram coletadas em intervalos regulares de tempo.

Determinação especfotométrica. Ao final, as amostras foram centrifugadas (25000Xg-rotor Ra-1M Kubota), 10.000 rpm, 5 min. Medidas espectrofotométricas foram realizadas a 550nm para determinar o grau de redução do ferrocitocromo c dos sobrenadantes. A curva de $\mathrm{O}_{2}{ }^{-}$foi obtida pela conversão dos valores de absorbância para nanomoles: [0] $=47,7$ x valor da absorbância x volume da amostra coletada ${ }^{12}$.

Análise estatística. Os dados foram expressos em média \pm desvio padrão. Analisou-se a diferença das médias dentro dos grupos com o teste tpareado. Entre os grupos, utilizou-se análise de variância (ANOVA) e quando esta revelou diferença significativa, utilizou-se 0 teste de Tukey. A significância foi definida para $\mathrm{p} \leq 0,05$. 


\section{RESULTAD0S}

Índice de aderência. 0 índice de aderência foi expresso em porcentagem (\%). Os resultados do IA de monócitos foram: do $\mathrm{CG}=67,3 \pm 14,5 \%$, do $\mathrm{AG}=69,2 \pm 9,7 \%$ e do $\mathrm{WAG}=66,3 \pm 13,4 \%$. Comparando-se as médias dos IA, não houve diferenças entre os grupos (ANOVA, $F=0,190 ; p=$ 0,827), Tabela 1.

Dosagem do TNF- $\alpha$. Os resultados da dosagem do TNFa foram:do CG $=135 \pm 51,6 \mathrm{pg} / \mathrm{mL}$, do $\mathrm{AG}=97 \pm 25,4 \mathrm{pg} /$ $\mathrm{mL}$ e do $\mathrm{WAG}=107 \pm .52,1 \mathrm{pg} / \mathrm{mL}$. Não houve diferença entre os grupos (ANOVA, $F=0,210 ; p=0,813$ ), Tabela 2 .

Tabela 1 - Comparação do índice de aderência (IA) de monócitos nos grupos de indivíduos sadios (CG) e pacientes esplenectomizados com autoimplante esplênico (AG) e sem auto-implante (WAG).

\begin{tabular}{lrr}
\hline Grupos & $\mathrm{N}^{\mathrm{p}}$ & Média $\pm \mathrm{DP}$ \\
\hline CG & 12 & $69,2 \pm 2,3 \%$ \\
AG & 18 & $67,3 \pm 4,2 \%$ \\
WAG & 9 & $66,3 \pm 4,4 \%$
\end{tabular}

$(p=0,827)$.

Tabela 2 - Comparação entre as concentrações de TNF- $\alpha$ em sobrenadantes de cultura de monócitos nos grupos de indivíduos sadios (CG) e pacientes esplenectomizados com auto-implante esplênico (AG) e sem autoimplante (WAG).

\begin{tabular}{lrr}
\hline Grupos & $\mathrm{N}^{\mathrm{p}}$ & Média $\pm \mathrm{DP}$ \\
\hline $\mathrm{CG}$ & 12 & $135 \pm 51,6 \mathrm{pg} / \mathrm{mL}$ \\
$\mathrm{AG}$ & 18 & $97 \pm 25,4 \mathrm{pg} / \mathrm{mL}$ \\
WAG & 9 & $107 \pm 52,1 \mathrm{pg} / \mathrm{mL}$ \\
\hline
\end{tabular}

$(p=0,813)$.

Liberação de $\mathbf{0}_{2}$ ' nos monócitos. № CG, houve aumento na produção média de $0_{2} \cdot$ em monócitos estimulados entre a $1^{\text {a }}$ e a $2^{\text {a }}$ h: com PMA $(14,5 \pm 4,5$ vs $20,8 \pm 6,2 ; p \leq 0,001$ teste $t$ pareado); com PMA + tuttsina (13,5 $\pm 5,1$ vs $23,2 \pm 8,6 ; p=$ 0,003 , teste t pareado). № WAG, houve diferença na produção média de 0 - entre a 1 a e a 2 a $h(4,2 \pm 2,2$ vs $7,1 \pm 2,3 ; p=$ 0,009 , teste t pareado); porém, nos monócitos estimulados com

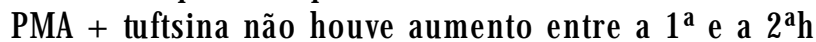
$(3,7 \pm 2,9$ vs $6,7 \pm 3,5 ; p=0,052$, teste t pareado). Aprodução média de $0_{2}$ - dos monócitos de $\mathrm{AG}$, estimulados com PMA, não aumentou entre a $1^{\mathrm{a}} \mathrm{e} 2^{\mathrm{a}} \mathrm{h}(14,9 \pm 7,3$ vs $16,1 \pm 5,6, \mathrm{p}=0,595)$ Figura 1. Contudo, houve aumento, entre a $1^{\mathrm{a}}$ e $2^{\mathrm{a}} \mathrm{h}$, quando estimulados com PMA + tuftsina $(13,1 \pm 5,6$ vs $18,3 \pm 8,5, p=0,011$, teste "t" pareado); Figura 2.

\section{DISCUSSÃ0}

0 baço é ponto importante de encontro entre a informação antigênica do sangue e 0 sistema imune, devido sua grande irrigação e posição central na corrente sangüínea ${ }^{28}$. Nas disfunções esplênicas, os micróbios circulam maior tempo no sangue, multiplicando-se em poucas horas ${ }^{9}$. No período precoce pósesplenectomia, defeitos da função antimicrobiana de fagócitos contribuem para bacteremia e mortalidade subseqüentes ${ }^{20}$.

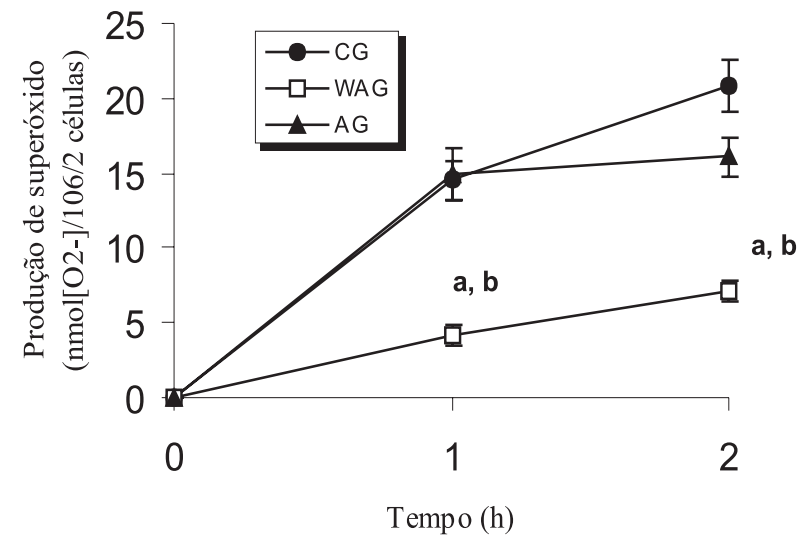

CG $(\bullet)$, AG $(\boldsymbol{\Delta})$ e WAG $(\square)$. a: diferença significativa entre WAG e CG ( $\mathrm{p} \leq 0.05$ da 1 ah para a 2 a $h) ; b$ : diferença significativa entre WAG e AG ( $p \leq 0.05$ na 1 ah e 2 h h).

Figura 1 - Dosagem de 0 - em sobrenadantes de cultura de monócitos estimulados com PMA às $0,1 \mathrm{e} 2 \mathrm{~h}$. 0 s resultados são representados em média \pm DP.

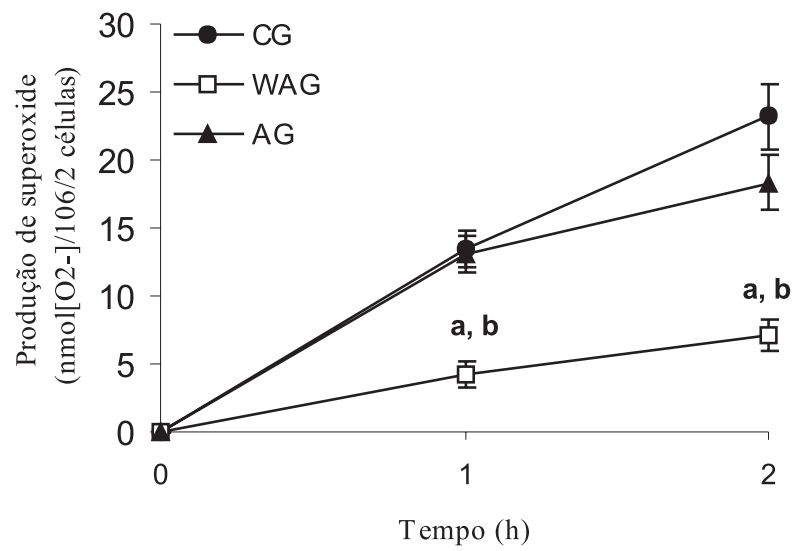

CG $(\bullet)$, AG $(\boldsymbol{\Delta})$ e WAG $(\square)$. a: diferença significativa entre WAG e CG ( $p \leq 0.05$ da $1^{\text {ah }}$ para a $2^{a}$ h) ; b: diferença significativa entre WAG e AG $(p \leq 0.05$ na $1^{\text {ah }}$ e $2^{\text {an }}$ ).

Figura 2 - Dosagem de $0_{2}$ - em sobrenadantes de cultura de monócitos estimulados com PMA + tuftsina às 0,1 e $2 \mathrm{~h}$. 0 s resultados são representados em média $\pm \mathrm{DP}$.

No presente estudo, 0 auto-implante esplênico foi realizado no grande epíploo. Este procedimento reduz a sepse pósesplenectomia em crianças $^{517}$. Contudo, a aderência e a produção de TNF- $\alpha$ não alteraram nos esquistossomóticos, pósesplenectomia, com ou sem auto-implante esplênico. Segundo Patel ${ }^{24}$, 0 grande epíploo é o local mais apropriado, em função da rica vascularização, permitindo o fluxo abundante de células inflamatórias, fatores de crescimento e citocinas. 0 baço contém grandes quantidades de linhagens mono-macrofágicas, envolvidas na endocitose e degradação de moléculas estranhas ${ }^{30}$. Estas células participam da resposta imune pela aderência a substratos; quimiotaxia; ingestão de células e partículas inertes; fagocitose de bactérias; liberação de mediadores bioativos, de citocinas próinflamatórias e de radicais livres derivados do oxigênio ${ }^{829}$.

A aderência é 0 passo inicial no processo fagocític ${ }^{26}$. Garraud et $\mathrm{a}^{10}$, usando modelo experimental de malária, concluíram que a esplenectomia, indicada na infecção malárica, não modifica a aderência monocítica. Aker et $\mathrm{al}^{1}$ concluíram não haver prejuízo da aderência celular em esplenectomizados 
com doença de Gaucher, na ausência esplênica pós-trauma ${ }^{1}$. Contudo, em pacientes com talassemia maior, pósesplenectomia, há aumento da aderência neutrofílica após 20 dias, voltando ao normal 90 dias após ${ }^{16}$. Já a aderência de macrófagos murinos, pós-esplenectomia reduz e se recupera após 0 auto-implante ${ }^{4}$.

Os fagócitos geram reativos microbicidas como $0 \mathrm{O}_{2}$. A sua liberação depende da densidade e do tipo de células, do estímulo e de outras condiç̃õe ${ }^{18}$. Picos de $\mathrm{O}_{2}$ - foram encontrados com $72 \mathrm{~h}$ e mensuráveis até 5 dias pós-estimulaçãa $0^{15}$. 0 pico de produção de $\mathrm{O}_{2}$ p por macrófagos estimulados com Enterococos faecalis, situa-se aos $60 \mathrm{~min}^{27}$. Em nosso estudo, a geração de $0_{2}$ pelos monócitos dos controles, foi crescente da $1^{\underline{a}}$ para a $2^{\underline{a}}$ hora, tanto nos monócitos estimulados com PMA como nos tratados com tuftsina. Este aumento confirma nossos resultados prévios sobre a curva de produção do $\mathrm{O}_{2}{ }^{-}$em ratos, estimulados com PMA, mostrando elevação crescente até as $6 \mathrm{~h}$ iniciais, com redução a 50\% do valor máximo após $24 \mathrm{~h}$, em seguida valores quase imensuráveis? ${ }^{7}$. No presente estudo, observamos também que os esplenectomizados têm níveis bastante reduzidos de $\mathrm{O}_{2}$, comparados aos controles ou auto-implantados, embora apresentem 0 padrão de aumento na liberação da $1^{\mathrm{a}}$ para a $2^{\mathrm{a}}$ h. Os monócitos dos auto-implantados liberaram tanto $\mathrm{O}_{2}{ }^{-}$quanto os controles e ambos mais que os com esplenectomia total sem auto-implante. Nos esplenectomizados, a tuftsina não alterou a liberação de $\mathrm{O}_{2}$ em monócitos. A esplenectomia poderia reduzir a tuftsina; porém, sua concentração estava normal nos pacientes que desenvolveram esplenose após lesões traumáticas do baço ${ }^{31}$. Concordando com os nossos dados, em outro estudo, esplenectomizados revelaram migração aleatória e aderência normal dos neutrófilos, porém produção de $\mathrm{O}_{2} \cdot \mathrm{e}$ de peróxido de hidrogênio prejudicadas ${ }^{16}$. A inoculação de polissacarídios da cápsula de pneumococos resulta em aumento na liberação de $0_{2}$ pelas células de Kupffer e dano das células endoteliais nos sinusóides ${ }^{19}$. Estas alterações são atenuadas pelo tratamento com SOD ou pela esplenectomia, assim, esta reduz a produção de $\mathrm{O}_{2}$, uma proteção contra a injúria hepática ${ }^{21}$. A função fagocítica de macrófagos diminui após esplenectomia parcial em ratos. Entretanto, o remanescente esplênico permanece com a capacidade de ser estimulado quando exposto a antígeno $0^{22}$. No presente estudo, não se observou aumento da $1^{\mathrm{a}}$ para a $2^{\underline{a}} \mathrm{~h}$ da produção de $\mathrm{O}_{2}$ nos monócitos dos pacientes autoimplantados pós-estímulo com PMA. Todavia, 0 acréscimo de tuftsina sintética à cultura dos monócitos resultou em aumento da produção de $\mathrm{O}_{2}$. A tuftsina estimula a atividade de fagócitos. Nos demonstramos que os níveis séricos de tuftsina de crianças com esplenose são semelhantes aos das saudáveis ${ }^{5}$.

A esplenectomia parece remover uma fonte importante de macrófagos/monócitos do local de processamento de reações imunes. A produção reduzida de $\mathrm{O}_{2}$ em esplenectomizados pode ser um dos fatores implicados na resposta deficiente do hospedeiro às infecções. 0 auto-implante esplênico pode contribuir de forma sinérgica ou isolada para a manutenção da atividade microbicida normal dos monócitos, recuperando e mantendo as reações imunes nos fragmentos auto-implantados. Esta função dos monócitos representaria um marcador do processo patológico durante a sepse pós-esplenectomia.

\section{REFERÊNCIAS BIBLIOGRÁFICAS}

1. Aker MZA, Abrahamov A, Horowitz MMY. Abnormal neutrophil chemotaxis in Gaucher disease. British Journal Haematology 83:187-191, 1993.

2. Babior, BM. Superoxide: a two-edged sword. Brazilian Journal of Medical and Biological Research 30: 141-155, 1997.

3. Barreto VST, Domingues Alce. Doenças hepáticas na esquistossomose. In: Coelho J (ed) Aparelho digestivo. Clínica e Cirurgia, Medsi. Rio de Janeiro, p.1071-1084, 1996.

4. Bowen TJ, Ochs HD, Altman LC, Price TH, Van Epps DE, Brautigan DI, Rosin RE, Perkins WD, Babior BM, Klebanoff SJ, Wedgwood RJ. Severe recurrent bacterial infections associated with defective adherence and chemotaxis in two patients with neutrophils deficient in a cell-associated glycoprotein. Journal of Pediatrics 101: 932-940,1982.

5. Brandt CT, Maciel DT, Caneca OAF, Castro CMMB, Araújo LB. Autotransplant of spleen tissue in children with schistosomiasis: evaluation of splenic function after splenosis. Memórias do Instituto Oswaldo Cruz 96: 117-22, 2001.

6. Cohn ZA. The activation of mononuclear phagocytes: fact, fancy, and future. Journal of Immunology 121: 813-816, 1978.

7. De Castro CMMB, Manhães de Castro R, Medeiros A; Santos AQ, Silva WTFE, Lima Filho JL. Effect of stress on the production of $\mathrm{O}_{2}$ in alveolar macrophages. Journal of Neuroimmunology 108: 68-72, 2000.

8. De La Fuente M, Del Rio M, Ferrandez MD, Hernanz A. Modulation of phagocytic function in murine peritoneal macrophages by bombesin, gastrin-releasing peptide and neuromedin C Immunology 73: 205-211, 1991

9. Diamond LK. Splenectomy in childhood and the hazard of overwhelming infection. Pediatrics 43: 886-889, 1969.

10. Garraud 0, Poingt JP, Perraut R, Gysin J. Peripheral blood mononuclear cell in the squirrel monkey Saimiri sciureus: characterization and functional aspects of T lymphocytes. Research in Immunology 140: 857 874, 1989.

11. Harding B, Kenny F, Given F, Murphy B, Lavelle S. Autotransplantation of splenic tissue after splenectomy in rats offers partial protection against intravenous pneumococal challenge. European Surgical Research 19:135139,1987

12. Johnston Jr RB. Measurement of $\mathrm{O}_{2}$ - secreted by monocytes and macrophages. Methods in enzimology 105:365-369, 1984.

13. Katz N, Peixoto SV. Análise crítica da estimativa do número de portadores de esquistossomose mansoni no Brasil. Revista da Sociedade Brasileira de Medicina Tropical 33:303-308, 2000.

14. Kays MA, Stolar CJH. The spleen and splenectomy: implication for the pediatric population. In Fkalsrud EW, Krumel TM. Infectious and immunologic disorders in pediatric surgery. W.B Saunders Company, Philaldelphia, 91-100, 1993.

15. Kelley JL, Rozek MM, Suenram CA, Schwartz CJ. Activation of human blood monocytes by adherence to tissue culture plastic surfaces. Experimental and Molecular Pathology 46: 266-278, 1987.

16. Lianou PE, Bassaris HP, Skoutelis AT, Votta EG, Papavassilou JT, Phair JP. Transient improvement of poltmorphonuclear leukocyte function by splenectomy in betathalassemia. Medical microbiology Immunology 176: 209-215,1987.

17. Lüdtke FE, Mack SC, Schuff-Werner P, Noth E. Splenic function after splenectomy for trauma. Role of autotransplantation and splenosis. Acta Chemica Scandinavica 155: 533-539, 1989.

18. Markert M, Andrews PC, Babior BM. Measurement of $\mathrm{O}_{2}$-production by human neutrophils. The preparation and assay of NADPH oxidasecontaining particles from human neutrophils. Methods in enzimology 105 : 358-365, 1984.

19. Matsuo S, Nakagawara A, Ikeda K, Mitsuyama M, Nomoto K. Enhanced release of reactive oxygen intermediates by immunologically ativated rat kupffer cells. Clinical Experimental Immunology 59: 203, 1985.

20. McCarthy JE, Redmond PH, Duggan-SM, Watson RW, Condron CM, O'Donnell JR, Bouchier Hayes DJ.Characterization of the defects in murine 
peritoneal macrophage function in the early postsplenectomy period.Journal of Immunology155:387-96, 1995.

21. Mizukami T, Yokoyama H, Okamura Y, Ohgo H, Fukuda M, Kamegaya Y, Kato S, Ishi H. Splenectomy attenuates superoxide anion release into the hepatic sinusoid after lipopolysaccharide challenge. Journal of Hepatology 31: 235-241, 1999.

22. Müftïoglu TMA, Köksal N, Özkutlu D. Evaluation of phagocytic function of macrophages in rats after partial splenectomy. American College of Surgery 191: 668-671, 2000.

23. Noga SJ, Normanm SJ, Winer RS. Methods in laboratory investigation isolation of guinea pig monocyte and Kurloff cells: characterization of monocyte subsets by morphology, cytochemistry and adherence. Laboratory Investigation 51: 244-252, 1984.

24. Patel JM, Williams JS, Shijel B, Hinshaw JR. Preservation of splenic function by autotransplantation of traumatized spleen in man. Surgery 90: 683-688, 1981.

25. Roos D, Winterbourn CC. Lethal Weapons. Science 296: 669-671, 2002.

26. Segura JJ, Jiménez-Rubio A. Effect of eugenol on macrophage adhesion in vitro to plastic surfaces. Journal Endodontic 24: 229-231, 1998.
27. Sübmuth SD, Muscoll-Silberhorn A, Wirth R, Susa M, Marre R, Rozdzinski E. Aggregation substance promotes adherence, phagocytosis and intracellular survival on Enterococcus faecalis within macrophages and supress respiratory burst. Infection and Immunity 68:4900-4906, 2000.

28. Times W, Leemans R. Splenic autotransplantation and the immune system. Annals of Surgery 215: 256-260, 1991.

29. Zapolska-Donar D, Naruszewicz M, Zapolska-Donar A, Markiewski M, Millo BB. Ibuprofen in hibits adhesiveness of monocytes to endothelium and reduces cellular oxidative stress in smokers and no-smokers. European Journal of Clinical Investigation 30: 1002-1010, 2000.

30. Zareie M, Singh PK, Irvine EJ, Sherman PM, Mckay DM, Perdue MH. Monocyte/macrophage activation by normal bacteria and bacterial products. Implications for altered epithelial function in Crohn's disease. American Journal of Pathology 158: 1101-1109, 2001.

31. Zoli G. Splenic autotransplantation after splenectomy: tuftisin activity correlates with residual splenic function. British Journal of Surgery 81: 716-718, 1994. 